\title{
Color Reproduction on Shrink Sleeves
}

\author{
Vahid Babaei ${ }^{(1)}$, Cédric Pelvet ${ }^{(2)}$ and Roger D. Hersch ${ }^{(1)}$ \\ (1) Ecole Polytechnique Fédérale de Lausanne, Lausanne, Switzerland
}

(2) Quadraxis SAS, Cherbourg, France

\begin{abstract}
Wrapping heat-deformable plastic labels around packages relies on a shrinking process. Shrinking plastic labels distorts not only the shape but also the color of the printed artwork. In this work, we analyze and model the color shifts induced by shrinkage. The ultimate goal is to generate full color images which after shrinking have colors as close as possible to the original colors. For this purpose, we present a thickness enhanced Clapper-Yule prediction model. Its calibration requires spectral measurements of original, non-shrunk samples as well as the measured shrinking factors. With the prediction model, we establish a table creating the correspondences between target colors after shrinking and ink area coverages. This enables creating color images which after shrinking match the original images.
\end{abstract}

Keywords: Color reproduction, shrink sleeve, material deformation, ink thickness, Clapper-Yule prediction model

\section{Introduction}

Artworks printed on packages have the important role of communicating a message to the consumer. Plastic is the leading substrate in the packaging industry ${ }^{1}$. Plastic heat-shrinkable sleeve labels, commonly called shrink sleeves, were originally developed in the 1970s and rapidly became ubiquitous. These heat-deformable labels are printed, tubed and, by being exposed to a hot medium, shrunk around the product. Shrink sleeves can be found on the store shelves for consumer goods such as beverages, foods and body-care products. The durability, flexibility, tamper resistance and $360^{\circ}$ method of decoration have made shrink sleeves a multi-billion dollar industry. ${ }^{2}$

Shrink sleeves can be printed with different printing technologies, such as flexography, gravure, offset and liquid electrophotography. The printed artwork is comprised of color images, designs and text. The inks are usually printed on an opaque white base.

Shrinking the plastic label induces severe distortions. These distortions influence two aspects of the artwork printed on the sleeve: shape and color. Since it is immediately visible, the problem of shape 
distortion of shrink sleeves is widely known. Graphics located within different areas of the sleeve undergo a high or low distortion depending on the shrinking degree of that area. One may predict the shape distortion and, based on this prediction, pre-distort the shape to compensate for the shrinking effect. Therefore, the artwork is warped so as to look geometrically accurate after shrinking.

A similar problem occurs with respect to the color of shrink sleeves. Depending on the shrinkage, colors may be subject to large deviations. The shift in color as a result of shrinking is often overlooked. However, in demanding areas such as cosmetics, high accuracy color reproduction on shrink sleeves is essential.

For high quality color reproduction, the color distortion needs to be characterized and compensated for. One may achieve a correct final color by color corrections relying on successive printing and measuring cycles. Such an approach is expensive and cumbersome especially due to the nonlinear relationship between the amount of inks and the resulting colors.

In the present contribution, we develop a framework for high fidelity color reproduction on shrink sleeves. We use a similar concept as for the shape distortion problem. Given as input the amount of inks as well as the shrinking factor, we try to predict the resulting color using an extension of the Clapper-Yule color prediction model that accounts for colorant thickness variations. Its calibration requires only spectral measurements of original, non-shrunk samples as well as the shrinking factors of the shrunk samples. Utilizing this predictive framework, we fit the amount of input inks needed to obtain after shrinking the desired printed color image. We tested the framework on color images printed with cyan, magenta and yellow inks.

When the shrinking degrees are moderate, this approach provides a good reproduction accuracy. At high shrinking degrees, as a result of our laboratory-scale printing and shrinking setup, there are wrinkle artifacts that reduce the precision of the proposed model. For a very accurate reproduction at high shrinking degrees, the original Clapper-Yule model can be used. Its calibration however requires spectral measurements at each selected shrinking degree.

Although the need for high quality color reproduction on shrink sleeves is mentioned in the literature $^{3}$, no solution is proposed. Golob et al. ${ }^{4}$ report about the color shifts of sleeves when shrinking on different substrates. In the textile industry, color distortion due to material deformation occurs when drawing and texturizing colored filaments. ${ }^{5}$ To the best of our knowledge, this is the first time that a mathematical model is established to quantify the effect of sleeve deformation on the resulting colors.

In Section 2, we study the color shifts of printed shrink sleeves for several degrees of shrinking. In Section 3 we propose a color prediction model for shrink sleeves that predicts the color of the sleeves 
after printing and shrinking. Section 4 describes the experimental setup for the printing and shrinking processes. Section 5 presents the accuracy of the proposed models in predicting the color of solid halftone patches for different shrinking factors. In Section 6 we describe the color reproduction workflow that enables printing color images which, after shrinking, are close to the original images.

\section{Color distortion due to shrinking}

In this section, we investigate the color distortions caused by shrinking the plastic sleeves. We define the shrinking factor as the ratio of the shrunk area of a color patch to its initial area. Figure 1 shows the reflectance factor of a solid cyan colorant after being shrunk to $75 \%, 61 \%$ and $45 \%$. Note that the higher the degree of shrinking, the higher the distortion. Sleeve shrinking thickens the corresponding substrate and ink layers. According to the Beer-Bouguer law ${ }^{6}$, a greater thickness results in a lower transmittance.

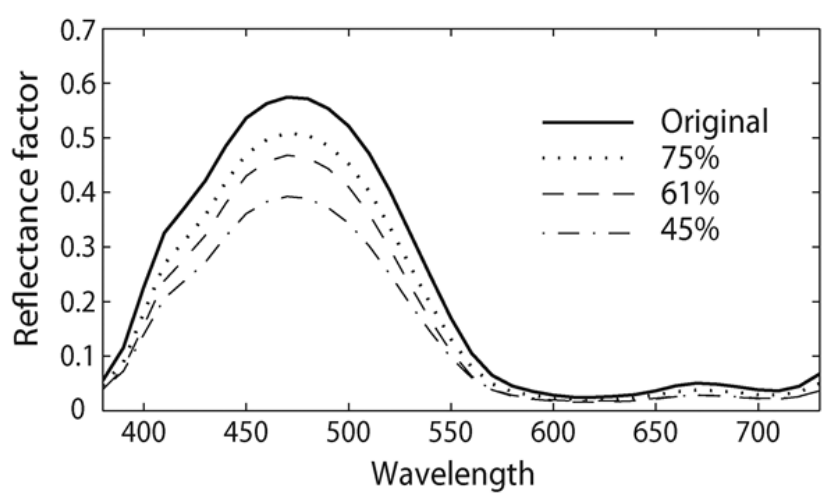

Figure 1. The reflectances of a cyan full-tone sample shrunk with different shrinking factors.

To examine how the colors of samples change after shrinking, we print representative color halftones on shrink sleeves and measure their reflectance factors. Then, we shrink the printed samples at several shrinking factors and measure their reflectance factors again (see Section 4 for details about the experiments). Table I provides the color shifts when shrinking a set of 125 color halftones, expressed as CIELAB $\Delta E_{00}$ color difference ${ }^{7}$.

Let us examine in detail the samples shrunk to $66 \%$. Figure 2 presents the color coordinates of some of these samples before and after shrinking in the CIELAB a* $\mathrm{L}^{*}$ and $\mathrm{a} * \mathrm{~b} *$ planes. Shrinking induces a significant decrease in lightness. The average CIELAB lightness of the 125 halftones under the D65 illuminant decreases from $L^{*}=58$ to $\mathrm{L}^{*}=53$. The darker the samples, the stronger their decrease in lightness. Among the 8 Neugebauer primaries formed by superpositions of CMY inks, the white diffuse substrate is the one with the least color change due to shrinkage. This is due to the fact that the white substrate has a very low absorption. Darker halftones are the ones with a smaller ratio of white 
colorant and are therefore more sensitive to shrinking. Shrinking also induces a slight increase in chroma (average $\Delta C^{*}=+1.1$ ). We observed that halftones with a significant amount of yellow ink and zero or a negligible amount of cyan undergo the largest chroma shifts.

Table I. The color shift between the colors of the non-shrunk samples and colors of the shrunk samples for 125 color halftones shrunk at different shrinking factors. The color shift is expressed as mean, $95 \%$ quantile and maximal CIELAB $\Delta E_{00}$ color difference under the D65 illuminant. The 125 halftones comprise all combinations of cyan, magenta and yellow inks at $0,25,50,75$ and $100 \%$ area coverages.

\begin{tabular}{cccc}
\hline shrinking factor & \multicolumn{3}{c}{$\Delta E_{00}$} \\
\cline { 2 - 4 } & mean & $95 \%$ & $\max$ \\
\hline $75 \%$ & 4.35 & 6.68 & 8.95 \\
$66 \% *$ & 4.84 & 7.35 & 8.13 \\
$61 \%$ & 7.51 & 11.31 & 12.51 \\
$45 \%$ & 10.20 & 14.48 & 16.83 \\
\hline
\end{tabular}

* At $66 \%$, the experiments were carried out using a slightly different setup with a 5-liter water pot heated slowly up to $70{ }^{\circ} \mathrm{C}$.
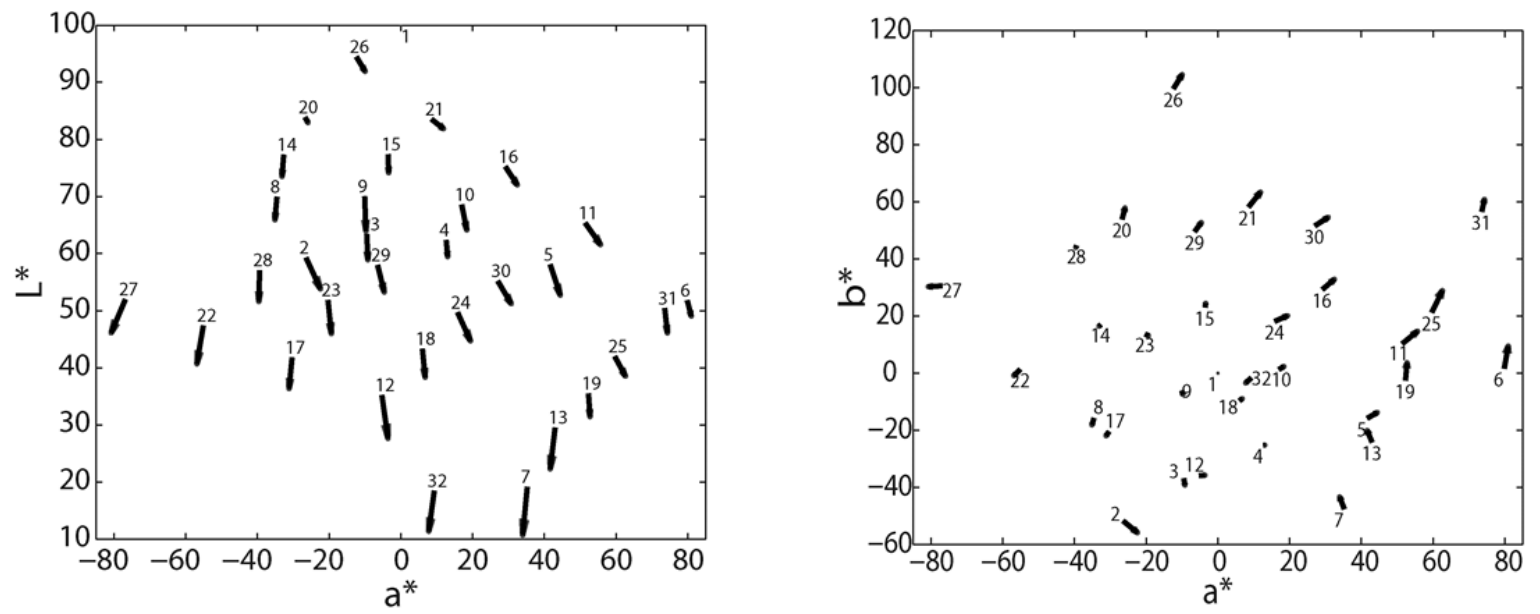

Figure 2. The change in CIELAB lightness and chroma (under the D65 illuminant) of color halftones after $66 \%$ shrinking. The arrows start at the color of the non-shrunk samples and point to their color after shrinking. Corresponding numbers are located close to the arrow tails.

\section{A spectral prediction model for shrink sleeves}

In order to compensate for color deviations induced by the shrinking process, we first need to predict the color of the printed and shrunk samples. For this purpose we use a spectral prediction model. It predicts the printed halftone color as a function of the area coverages of the inks. The prediction model requires a calibration step requiring a few dozens of spectral measurements ${ }^{8,9}$. In this section, we develop a spectral prediction model for shrink sleeves. It takes as input the nominal amount of inks as well as the shrinking factor and calculates the resulting color.

A straightforward approach for establishing a color prediction model for shrink sleeves consists in 
calibrating the model directly with the shrunk samples. We may measure the reflectances of the calibration patches at several shrinking factors. Based on the spectral measurements made for the selected shrinking factors, we can build the shrunk-sample calibrated color prediction model that relies on a model calibrated separately at each selected shrinking factor. However, this approach requires a significant number of spectral measurements.

Alternatively, we can build a prediction model by relying only on the calibration of the original, undistorted samples. We make the following simplifying assumptions. (1) Since the inks are nonscattering, we assume that Beer's law is valid. (2) We assume that shrinking preserves the ink volume. Therefore, shrinking the surface of the ink layer results in an inversely proportional, uniform increase of the ink layer thickness. (3) The hot water treatment does not change the sample's inherent physical properties, e.g. its index of refraction. (4) All Neugebauer primaries in a halftone suffer equally from shrinking. (5) The relative halftone area coverages remain identical for the non-shrunk and shrunk samples. (6) We also verified that the water does not solve or remove the inks.

From the first and second assumptions, we can deduce that the transmittance of the ink is the original ink transmittance raised to the power of the relative thickness. The relative thickness is calculated by measuring the area of the original patch and dividing it by the measured area of the shrunk patch.

Among the classical color prediction models, the Clapper-Yule model ${ }^{10}$ accounts explicitly for the interaction between the light and the halftone prints. This model keeps track of light scattering and reflection within the substrate and of the multiple light reflections between the substrate and the printair interface. For a single ink halftone, the well-known Clapper-Yule expression is

$$
R(\lambda)=K r_{S}+\frac{\left(1-r_{S}\right) r_{g}(\lambda)\left(1-r_{i}\right)(1-a+a t(\lambda))^{2}}{1-r_{g}(\lambda) r_{i}\left(1-a+a t^{2}(\lambda)\right)},
$$

where $K$ gives the fraction of specularly reflected light reaching the spectrophotometer, $r_{s}$ is the specular reflection at the air-print interface, $r_{g}(\lambda)$ is the diffuse reflection by the substrate, $r_{i}$ represents the Fresnel reflectivity integrated over all incident angles at the print-air interface, $a$ is the fractional ink area coverage printed on the substrate and $t(\lambda)$ is the ink transmittance (see the Appendix for the derivation). Both the specular reflection $r_{s}$ and the internal reflection $r_{i}$ at the print-air interface depend on the refraction indices. According to the Fresnel equations for collimated light at an incident angle of $45^{\circ}$ and for a print surface having an index of refraction of 1.5 , the specular reflection factor $r_{s}$ is 0.04 . For the internal reflectance $r_{i}$ we use the reported value of $0.6^{6}$. Since in this work we use a $0^{\circ}: 45^{\circ}$ measuring geometry, the specular reflectance is discarded, i.e. $K=0$.

In order to deduce the intrinsic reflectance spectrum $r_{g}(\lambda)$ from Equation (1) we may set the ink cov- 
erage $a=0$ and measure the unprinted substrate reflectance $R_{w}(\lambda)$

$$
r_{g}(\lambda)=\frac{R_{w}(\lambda)-K r_{S}}{1+(1-K) r_{i} r_{S}+r_{i} R_{w}(\lambda)-r_{S}-r_{i}} .
$$

We then extract the transmittance $t(\lambda)$ of the colorant, by inserting into Equation (1) as $R(\lambda)$ the corresponding measured solid (100\%) colorant reflectance $R_{i}(\lambda)$ and by setting the ink area coverage $a=1$,

$$
t_{i}(\lambda)=\sqrt{\frac{R_{i}(\lambda)-K r_{S}}{r_{g}(\lambda) r_{i}\left(R_{i}(\lambda)-K r_{S}\right)+r_{g}(\lambda)\left(1-r_{i}\right)\left(1-r_{S}\right)}} .
$$

According to Beer's law, when increasing the thickness of an ink of transmittance $t(\lambda)$ by a factor $d$, its transmittance becomes $t(\lambda)^{d}$. By inserting the relative thickness $d$ as the power of the transmittance into the Clapper-Yule model (Equation (1)) and extending it to multi-ink prints we predict the reflectances of shrunk halftones having a relative thickness $d$

$$
R(\lambda)=K r_{S}+\frac{\left(1-r_{S}\right) r_{g}(\lambda)\left(1-r_{i}\right)\left(\sum_{j=1}^{m} a_{j} t_{j}^{d}(\lambda)\right)^{2}}{1-r_{g}(\lambda) r_{i} \sum_{j=1}^{m} a_{j}\left(t_{j}^{d}(\lambda)\right)^{2}},
$$

where $a_{j}$ are the area coverages and $t_{j}(\lambda)$ the transmittances of the contributing colorants, also called Neugebauer primaries. A similar but more complex model has been used in a previous work to deduce ink thickness variations from color halftone prints in order to regulate the ink flow variations in an offset press ${ }^{11}$. That model dealt with the inverse problem of recovering ink thickness variations from color halftone images. Spectral variations of colorants were expressed as individual thickness variations of each of three contributing inks. In the present model, the thickness variation model is much simpler, since all colorants are made thicker by a single thickness increase factor $d$.

The prediction accuracy of the Clapper-Yule model depends on the accuracy of our estimate of the effective area coverage $a_{j}$ of the contributing colorants. In this work, we use the superposition dependent ink spreading model proposed by Hersch et al. ${ }^{12}$ to calculate effective area coverages. In classical tone reproduction models, there is one curve mapping nominal to effective area coverages for each single ink. In contrast, the superposition dependent ink spreading model accounts also for the interaction between an ink halftone and the other superposed inks. It relies on the fact that ink spreading is generally different when printing a halftone in superposition with paper or in superposition with another ink. 
In case of three inks $c, m$ and $y$, instead of having only one ink spreading curve per ink, we have one ink spreading curve per ink and per superposition condition. A superposition condition is defined as the superposition of a halftoned ink with paper $i$, with one solid ink $i / j$ or with two solid inks $i / j k$. We obtain 12 superposition conditions yielding 12 ink spreading curves $(c, m, y, c / m, c / y, m / c, m / y, y / c$, $y / m, c / m y, m / c y$ and $y / c m)$. With halftone nominal area coverages of $0.25,0.5$ and 0.75 we have three halftone patches for characterizing each ink spreading curve. The calibration set comprise therefore 36 patches for the 12 ink spreading curves plus the 8 Neugebauer primaries, yielding a total of 44 patches.

After calibrating the model, one obtains the effective area coverages of a given color halftone by weighting the ink spreading curves according to the underlying area coverages of the colorants forming that halftone. ${ }^{12}$ Hereinafter, we call "standard Clapper-Yule" the classical Clapper-Yule model with effective area coverages calculated by accounting for superposition dependent ink spreading. We call "thickness enhanced Clapper-Yule" the Clapper-Yule model with the relative colorant thickness factor which also accounts for superposition dependent ink spreading.

\section{Experiments}

We designed experiments to verify the validity of the thickness enhanced Clapper-Yule model for predicting the reflectance of shrunk samples. We also produced full color images that are conceived so as to have the desired colors after shrinking (Section 6).

All samples were printed with an HP Indigo WS6000 Digital Press. The substrate is a transparent Polyethylene Terephthalate Glycol $50 \mu \mathrm{m}$ thick film with a main shrinkable orientation in traverse direction (TD). We use mutually rotated clustered-dot cyan, magenta and yellow halftones at a screen dot frequency of 180 lpi and a printer resolution of 812 dpi. A set composed of 125 representative halftone patches $(13 \mathrm{~mm} \times 40 \mathrm{~mm}$ each $)$ is printed using all combinations of cyan, magenta and yellow at $0,25,50,75$ and $100 \%$ area coverages. This set includes both calibration and test patches. A diffusing white layer is printed on top of the ink layers as a reflecting support. Figure 3 shows the order of the transparent plastic film, the inks and the diffusing white.

Despite the presence of a white diffusing ink, the prints are not completely opaque. To measure their reflectance spectra, we place them on top of a white background (see Figure 3). The reflectances are gathered using a Datacolor MF45 portable spectrophotometer with a $0^{\circ}: 45^{\circ}$ geometry. 


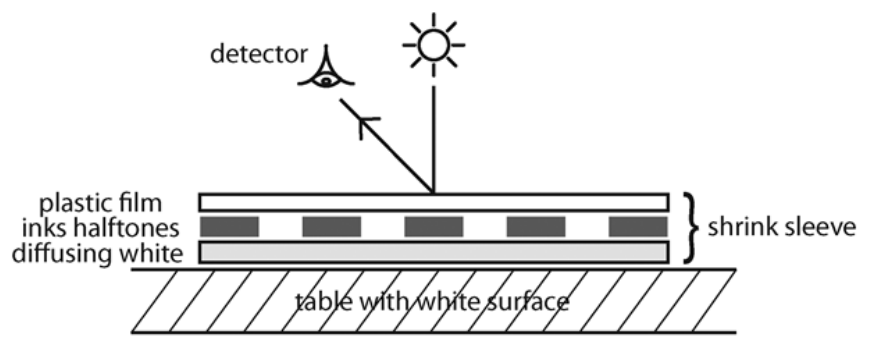

Figure 3. Layout of the layers forming the shrink sleeve, with reflectances measured according to the $0^{\circ}: 45^{\circ}$ geometry.

In order to shrink the samples we use a simple shrinking setup. We place an aluminum cylinder inside a 25 -liter pot of hot water. We form a cylinder with the printed film having its main shrinkable direction orthogonal to its axis. We place the cylindrical shrink sleeve around a smaller aluminum cylinder. After allowing the sleeve to remain about 15 seconds inside the $75^{\circ} \mathrm{C}$ water, the cylindrical label shrinks and fits the metal (see Figure 4). In order to prevent further shrinking of the labels, we put them in a pot of tap water for half a minute. Then, we remove them from the cylinders.

Note that the sleeves are also slightly shrunk along the machine direction (MD). Hence, we defined the shrinking factor as the ratio of halftone patch area before and after shrinking. Therefore, the shrinking factor is the multiplication of the machine direction and traverse direction shrinking sub-factors. In order to generate different shrinking factors, we use cylinders of different diameters yielding approximately shrinking factors of $75 \%, 61 \%$ and $45 \%$.
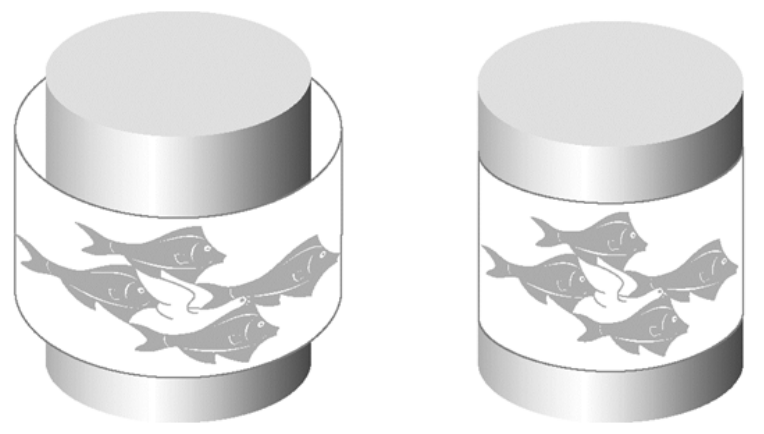

Figure 4. Our logo on a sleeve before and after shrinking using the described setup.

\section{Color prediction accuracy of shrink sleeves}

We first calibrate the thickness enhanced Clapper-Yule model with the reflectances of 44 non-shrunk samples. We then predict the reflectances of shrunk labels by measuring the shrinking factor of the test patches and increasing the ink thicknesses by the inverse of the shrinking factor in the thickness enhanced Clapper-Yule model (Equation (4)). Table II gives the prediction accuracy of this model expressed as CIELAB $\Delta E_{00}$ color difference ${ }^{7}$. The obtained prediction accuracy shows that our ap- 
proach of using the thickness modified transmittance is successful, especially for low and moderate shrinking factors. The first row in Table II is merely the prediction accuracy of the standard ClapperYule model without thickness modification for the test set comprising the 125 representative halftones, which includes the 44 reflectances used for calibrating the ink spreading curves and obtaining the Neugebauer primaries. The last three rows of Table II present the model accuracy for 125 halftones shrunk at three different shrinking factors. For these sets, we use the ink transmittances as well as the ink spreading curves acquired from the previously measured 44 non-shrunk calibration samples. We only increase the relative thicknesses of the inks by raising their transmittances with an exponent that is the inverse of the measured shrinking factor.

Table II. The color difference between the colors predicted by the thickness enhanced Clapper-Yule model and the measured colors for 125 color halftones shrunk with different factors under the D65 illuminant. The results rely only on the calibration of 44 non-shrunk samples and on the shrinking factors.

\begin{tabular}{cccc}
\hline shrinking factor & \multicolumn{3}{c}{$\Delta E_{00}$} \\
\cline { 2 - 4 } & mean & $95 \%$ & $\max$ \\
\hline $100 \%$ & 0.72 & 1.52 & 2.37 \\
$75 \%$ & 1.53 & 2.58 & 3.34 \\
$61 \%$ & 2.74 & 5.39 & 7.55 \\
$45 \%$ & 5.11 & 8.39 & 11.92 \\
\hline
\end{tabular}

Table II shows that with higher shrinking degrees the prediction accuracies tend to be less precise. To investigate the reason, we compare in Figure 5 the microscopic structures of a sample halftone of the same original color at three shrinking factors. At higher shrinking degrees, the samples show microwrinkles induced by the severe deformations. This effect partly invalidates the second simplifying assumption of the thickness enhanced Clapper-Yule model stating that the reduction in surface area is inversely proportional to the increase in ink thickness.
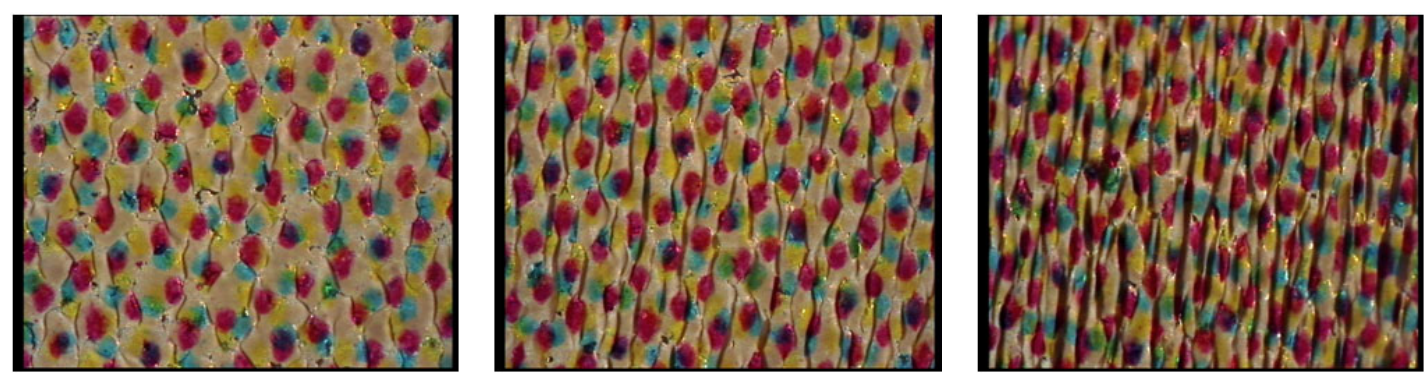

Figure 5. The microscopic image of a halftone shrunk to $75 \%, 61 \%$ and $45 \%$ from left to right, respectively (magnification: $40 \times$ ).

Further scrutinizing the samples reveals that only the ink layer shrinks non-uniformly and forms a fuzzy microstructure while the polymer substrate shrinks uniformly. This is not surprising since the employed HP Indigo Digital Press inks used for printing the samples have not been conceived for high shrinking degrees. In industrial gravure or flexography printing technologies, there are inks which 
shrink uniformly along with the substrate ${ }^{13}$. Also, a shrinking setup made of a steam tunnel instead of hot water enables uniform shrinking. This can be readily verified by looking under a microscope at the sleeves of drink bottles. Wrinkles are a side-effect of our "laboratory-scale" experimental setup. However, carrying out trials with industrial equipment is prohibitively expensive.

To reach a higher prediction accuracy for the samples having an unordered microstructure, we can use as model predicting printing and shrinking the shrunk-sample calibrated prediction model described at the beginning of Section 3. It consists of a standard Clapper-Yule model (Equation (7) in Appendix) requiring 44 calibration samples per selected shrinking factor. Table III presents the accuracy obtained with the Clapper-Yule model calibrated separately on 44 shrunk halftones at each shrinking factor. In Table III we consider two different test sets: (a) the test set comprising all 125 halftones including the 44 calibration samples and (b) the test set excluding the 44 calibration samples used to calibrate the model at each shrinking factor. No thickness modification is performed. This procedure offers excellent prediction accuracy at the expense of more spectral measurements.

Table III. The color difference between the colors predicted by the standard Clapper-Yule model and the measured colors of the samples under the D65 illuminant. At each shrinking factor, the model is separately calibrated with 44 samples and tested (a) on all 125 halftones and (b) on 81 halftones after excluding the calibration samples.

\begin{tabular}{lcccc}
\hline \multicolumn{2}{l}{ shrinking factor } & \multicolumn{3}{c}{$\Delta E_{00}$} \\
\cline { 3 - 5 } & & mean & $95 \%$ & $\max$ \\
\hline \multirow{2}{*}{$100 \%$} & $a$ & 0.72 & 1.52 & 2.37 \\
& $b$ & 0.90 & 1.65 & 2.37 \\
$75 \%$ & $a$ & 0.97 & 2.32 & 3.84 \\
& $b$ & 1.27 & 2.66 & 3.84 \\
$61 \%$ & $a$ & 1.59 & 3.36 & 4.25 \\
& $b$ & 1.89 & 3.60 & 4.25 \\
$45 \%$ & $a$ & 1.13 & 2.59 & 4.24 \\
& $b$ & 1.39 & 2.76 & 4.24 \\
\hline
\end{tabular}

\section{Color reproduction workflow for shrink sleeves}

The goal of the present work is to create accurately reproduced color images on shrink sleeves. To this end, we establish a color reproduction workflow ${ }^{14}$ taking into account both printing and shrinking. As in any color reproduction workflow, we first convert input colors from a source color space such as sRGB to a device independent color space such as CIELAB ${ }^{15}$. By performing gamut mapping ${ }^{16}$, we map the input colors into the narrower gamut formed by colors of the printed samples. We then carry out the color separation by converting the resulting printable colors into amounts of printer inks. The color separations are halftoned ${ }^{17}$ and printed. 
In this work, we implement the color reproduction workflow in two phases (Figure 6). The "preparation" phase aims at creating a gamut mapping table that maps the input sRGB gamut into the color gamut of the print samples. We also build the ink separation tables that establish the correspondences between desired printed and shrunk colors and the ink area coverages. During the "image reproduction" phase, input image colors are gamut mapped and color separated by a fast access to the tables created in the preparation phase.

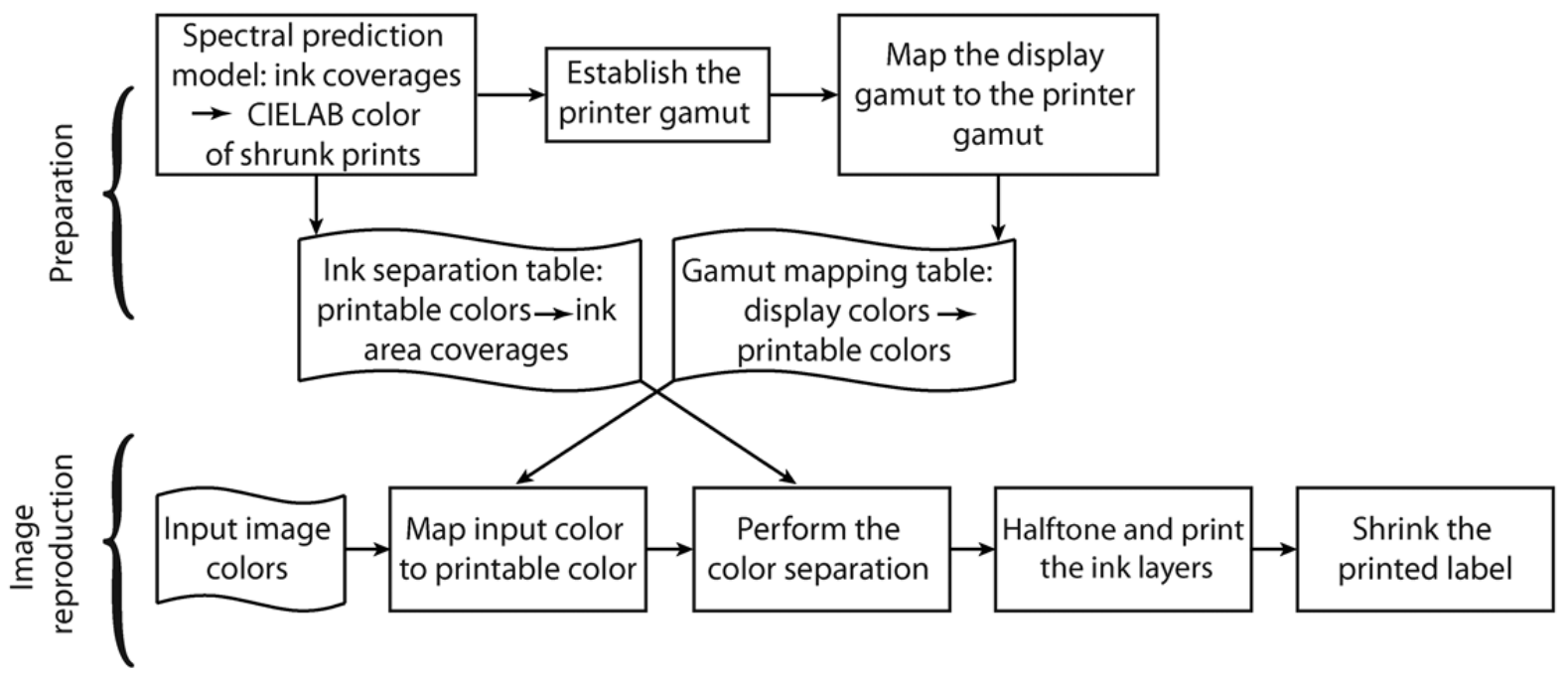

Figure 6. The color reproduction workflow composed of a preparation phase and of an image reproduction

phase.

Let us describe our approach in a more detailed manner. We set the target gamut as the gamut of the non-shrunk prints. This enables prints at different shrinking degrees to have the same gamut. This gamut is created with the standard Clapper-Yule model by varying the nominal ink area coverages of cyan, magenta and yellow in small intervals, predicting their reflectance spectra, converting them to CIE-XYZ tri-stimulus values under the D65 illuminant and computing the corresponding CIELAB colors. The target gamut is formed by the concave hull of these CIELAB colors ${ }^{18}$. We then build the gamut mapping table by mapping the sRGB colors into the target printer gamut ${ }^{16}$.

We produce one ink separation table per shrinking degree. For a specific shrinking factor, the ink separation table provides the area coverages of the inks that yield a given color after printing and shrinking. The area coverages of inks are found using the thickness enhanced Clapper-Yule model (Equation (4)), by setting the relative thickness $d$ as the inverse of the given shrinking factor. Ink area coverages are computed by minimizing for each table entry the $\Delta E_{94}$ difference between the predicted 
color and the desired color. The resulting ink separation table has $44 \times 81 \times 78$ entries obtained by steps of 2 sampling the $\mathrm{L}^{*}, \mathrm{a}^{*}$ and $\mathrm{b}^{*}$ axes within the non-shrunk, target gamut. Area coverages of intermediate colors are interpolated at color separation time.
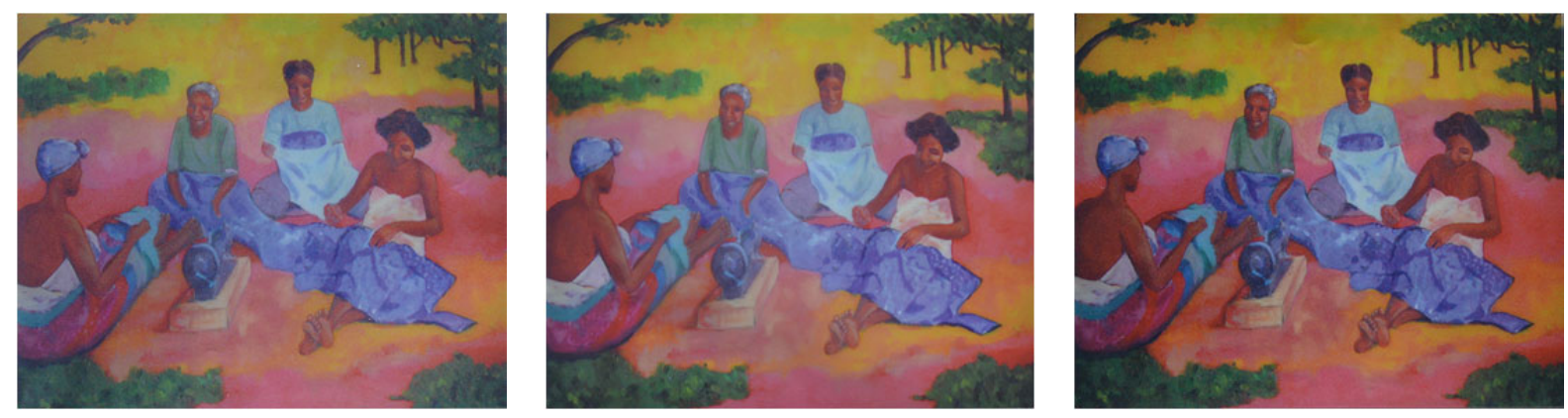

Figure 7. Center: the photograph of an original print without deformation. Left: the printed image after $61 \%$ shrinking created with our custom color reproduction framework. Right: the printed image after $61 \%$ shrinkage without any compensation.
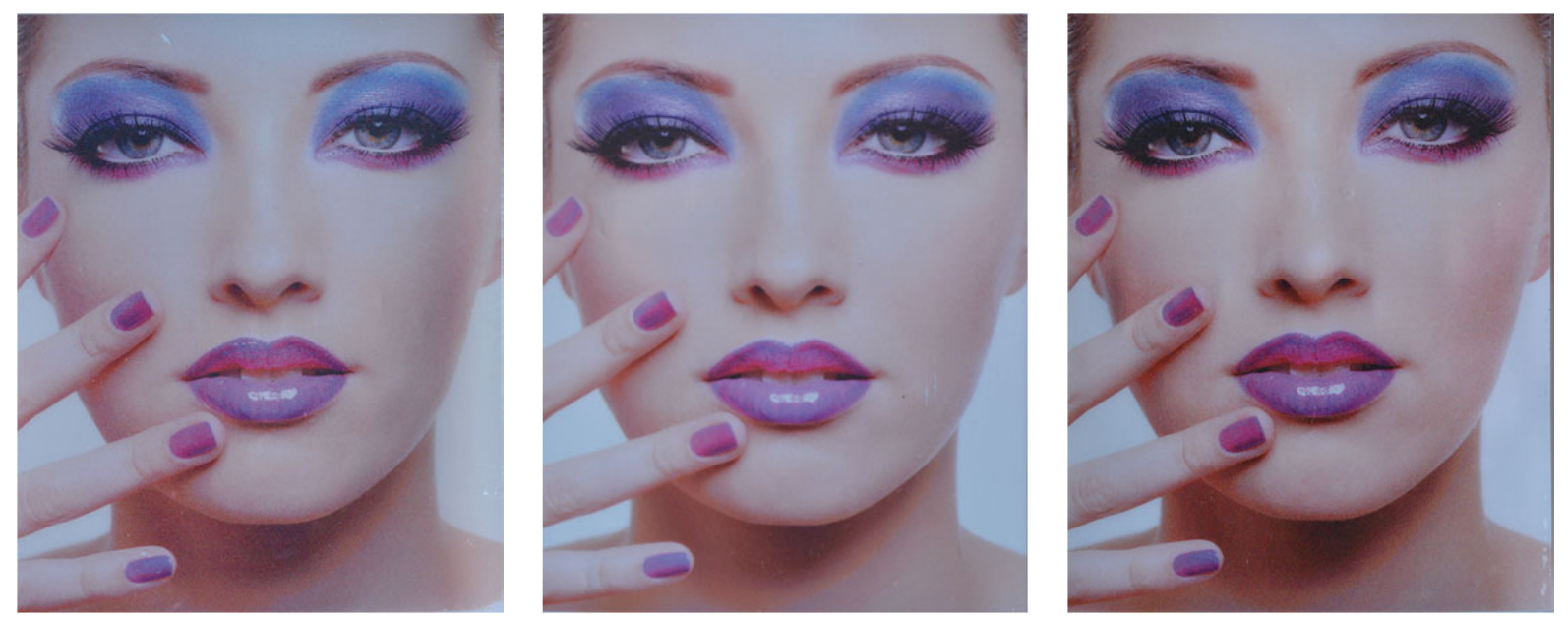

Figure 8. Center: the photograph of an original print without deformation. Left: the printed image after $45 \%$ shrinking created with our custom color reproduction framework. Right: the printed image after $45 \%$ shrinkage without any compensation.

Let us illustrate the proposed method with two representative color images that are shrunk with a given shrinking factor. In order to have the same image size after shrinking, we enlarge the image along the main shrinkable direction in proportion to the inverse of the shrinking factor. For image reproduction, the image colors are mapped into the gamut of the target printer using the gamut mapping table. Then, the color separations are generated by accessing the ink separation table associated with the considered shrinking factor. Halftoning, printing and shrinking are then carried out using the same procedure as for the other experiments. 
We take photos of the original, non-shrunk and of the shrunk printed images by a camera under the same acquisition conditions. The photos were taken by a DSLR Nikon D5200 with an exposure time of $1 / 50 \mathrm{~s}$ and an aperture of $\mathrm{f} / 5.6$. Figures 7 and 8 show that the images created by our thickness enhanced Clapper-Yule model look similar to the original, non-shrunk image. The prints without color compensation have an objectionable reduced lightness and an increased chroma mainly in the redyellow tones.

\section{Conclusion}

High quality color reproduction on heat shrinkable sleeves is a challenging task due to the deformation of the print. We apply a thickness enhanced Clapper-Yule model for predicting the reflectance spectra of shrunk halftones. We modify the colorant transmittances according to the measured shrinking factor and achieve a relatively good color prediction accuracy. The thickness-enhanced Clapper-Yule model requires for its calibration only 44 original, non-shrunk samples. With the thickness-enhanced Clapper-Yule model, we create for the target shrinking factor a table establishing the correspondence between desired colors and fitted ink area coverages. This yields color images which after shrinking resemble the originals.

At high degrees of shrinking, the color predictions offered by the thickness enhanced Clapper-Yule model are less accurate. This is due to the inks that form fuzzy micro-wrinkles. If very accurate color prediction at high shrinking degrees is desired, one can rely on the standard Clapper-Yule model with one set of calibration patches per selected shrinking factor.

In the future, it would be interesting to verify the accuracy of the proposed approach on labels that are printed and shrunk using professional equipment ${ }^{3}$. In this contribution, we dealt with cases having a single shrinking factor for the whole image. We may extend the software to process images with spatially variable shrinking factors. Future research may also include other forms of deformation of the sleeve labels such as stretching. Stretching may require adapting both the colors and the halftone screen frequency to the stretching factor.

\section{Acknowledgements}

We thank Raynald Cordey from Ecole Professionnelle Lausanne for fabricating the metallic cylinders. We thank the Swiss National Science Foundation for partly supporting this project with grant 200021-143501. 


\section{References}

[1] Kirwan MJ, Plant S, Strawbridge W. Plastics in food packaging. In: Coles R, Kirwan M, editors. Food and Beverage Packaging Technology, 2nd ed. Wiley-Blackwell; 2011. p. 157-161.

[2] Moore L. Technologies \& techniques, shrink sleeve labels take market by storm. Flexo 2012; 37: $50-59$.

[3] Vainstein J. A study of the conditions and variables that affect the printing of shrink films on waterbased flexography. M.Sc. thesis, Rochester Institute of Technology, 2005. https://ritdml.rit.edu/bitstream/handle/1850/1107/JVainsteinThesis2005.pdf?sequence=8

[4] Golob G, Svetec DG, Đordevic D, Žoroz B. Colour differences on offset printed pet and pvc shrink sleeve labels. Proceedings of the 34th International IARIGAI Research Conference 2007; 34: 251256.

[5] Amani Tehran M, Azimi B, Mojtahedi MR. Investigating the effect of false twist texturing process on the color coordinates variation of spun-dyed polyester filament yarns. Journal of Engineered Fabrics \& Fibers 2011; 6: 54-62.

[6] Emmel P. Physical models for color prediction. In: Sharma G, editor. Digital Color Imaging Handbook, CRC Press; 2003. p 173-181.

[7] Sharma G, Wu W, Dalal EN. The CIEDE2000 color-difference formula: implementation notes, supplementary test data, and mathematical observations. Color Res Appl 2005; 30 (1): 21-30.

[8] Balasubramanian R. Optimization of the spectral Neugebauer model for printer characterization. Journal of Electronic Imaging 1999; 8: 156-166.

[9] Hébert M, Hersch RD. Review of spectral reflectance models for halftone prints: Principles, Calibration, and prediction accuracy. Color Res Appl, appeared online, 2014.

[10] Clapper F, Yule J. The effect of multiple internal reflections on the densities of half-tone prints on paper. JOSA 1953; 43 (7): 600-603.

[11] Hersch RD, Brichon M, Bugnon T, Amrhyn P, Crété F, Mourad S, Janser H, Jiang Y, Riepenhoff M. Deducing ink thickness variations by a spectral prediction model. Color Res Appl 2009; 34: $432-442$.

[12] Hersch RD, Emmel P, Collaud F, Crété F. Spectral reflection and dot surface prediction models for color halftone prints. Journal of Electronic Imaging 2005; 14(3): 033001-0330012. 
[13] Pekarovicova V, Pekarovicova A, Fleming III PD. Shrink sleeve flexo inks. In: TAGA Proceedings 1998; 409.

[14] Morovic J, Lammens J. Color management. In: Schanda J, editor. Colorimetry: Understanding the CIE System, John Wiley; 2007. p 159-206.

[15] Wyszecki G, Stiles WS. Color Science. 2nd ed. New York: Wiley; 1982. p 130-175.

[16] Morovic J, Luo MR. The fundamentals of gamut mapping: A survey. Journal of Imaging Science and Technology 2001; 45(3): 283-290.

[17] Baqai FA, Lee JH, Agar AU, Allebach JP. Digital color halftoning. Signal Processing Magazine, IEEE 2005; 22 (1): 87-96.

[18] Bernardini F, Mittleman J, Rushmeier H, Silva C, Taubin G. The ball-pivoting algorithm for surface reconstruction, IEEE Trans.Visualization and Computer Graphics, 1999; 5 (4): 349-359.

\section{Appendix: Derivation of the Clapper-Yule formula}

Consider a single halftone layer with area coverage $a$ printed on a substrate (Figure 9). Incident light has the probability $a$ of reaching the substrate by passing through ink of transmittance $t(\lambda)$ and a probability $(1-a)$ of reaching the substrate without traversing the ink layer. Since $r_{s}$ is the specular reflection at the air-print interface, only portion $\left(1-r_{s}\right)$ enters the substrate. The light reaching the paper substrate is attenuated by a factor $\left(1-r_{s}\right)(1-a+a t(\lambda))$, with $(1-a+a t(\lambda))$ representing the attenuation of light by passing once through the halftone ink layer. Light is then laterally scattered and diffusely reflected by the substrate according to the substrate reflectance $r_{g}(\lambda)$. Traveling upwards, it traverses the print with a portion $a$ traversing the ink and a portion $(1-a)$ traversing an area free of ink. It is reflected at the print-air interface according to a reflection factor $r_{i}$, representing the Fresnel reflectivity integrated over all incident angles. The non-reflected part $\left(1-r_{i}\right)$ of the light exits. At the first exit, the spectral attenuation of the incident light is therefore $\left(1-r_{s}\right) r_{g}\left(1-r_{i}\right)(1-a+a t(\lambda))^{2}$. The part reflected at the print-air interface travels downward, is diffusely reflected by the substrate and travels upwards again. At the second exit, the spectral attenuation is $\left(1-r_{s}\right) r_{g}\left(1-r_{i}\right)(1-a+a t(\lambda))^{2} r_{i} r_{g}\left(1-a+a t^{2}(\lambda)\right)$.

By considering the light emerging after $0,1,2 \ldots, n-1$ internal reflections (Figure 9), we obtain the reflectance

$$
\begin{aligned}
& R(\lambda)=K \cdot r_{S}+\left(1-r_{S}\right) \cdot r_{g}\left(1-r_{i}\right) \cdot(1-a+a \cdot t(\lambda))^{2} \\
& \cdot\left(1+r_{i} \cdot r_{g}\left(1-a+a \cdot t^{2}(\lambda)\right)+\left(r_{i} \cdot r_{g}\left(1-a+a \cdot t^{2}(\lambda)\right)\right)^{2}\right. \\
& \left.\ldots+\left(r_{i} \cdot r_{g}\left(1-a+a \cdot t^{2}(\lambda)\right)\right)^{n-1}\right)
\end{aligned}
$$


where $K$ is the fraction of specularly reflected light reaching the spectrophotometer. For an infinite number of emergences, Equation (5) yields a geometric series. We obtain the well-known ClapperYule expression

$$
R(\lambda)=K r_{S}+\frac{\left(1-r_{S}\right) r_{g}(\lambda)\left(1-r_{i}\right)(1-a+a t(\lambda))^{2}}{1-r_{g}(\lambda) r_{i}\left(1-a+a t^{2}(\lambda)\right)}
$$

When specular reflectance is excluded from measurements, $K=0$. We obtain for a color patch printed with $m$ colorants

$$
R(\lambda)=K r_{S}+\frac{\left(1-r_{S}\right) r_{g}(\lambda)\left(1-r_{i}\right)\left(\sum_{j=1}^{m} a_{j} t_{j}(\lambda)\right)^{2}}{1-r_{g}(\lambda) r_{i} \sum_{j=1}^{m} a_{j}\left(t_{j}(\lambda)\right)^{2}} .
$$

where the sum of area coverages $a_{j}$ of the contributing colorants is 1 .

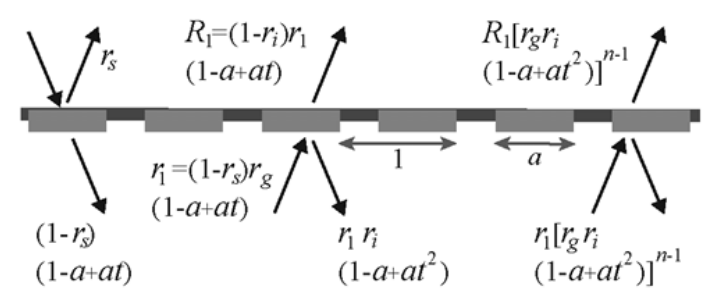

Figure 9. Attenuation of light by multiple reflections on a halftone printed patch. 